\title{
Tratamento Endodôntico em Pacientes Submetidos a Radioterapia: Revisão de
}

\section{Literatura}

\author{
Endodontic Treatment in Patients Under Radiotherapy Treatment: Literature Review \\ Tratamiento De Endodoncia En Pacientes Sometidos A Radioterapia: Revisión De La Literatura
}

Recebido: 15/05/2021 | Revisado: 23/05/2021 | Aceito: 28/05/2021 | Publicado: 11/06/2021

\author{
Diogo Alves de Araújo \\ ORCID: https://orcid.org/0000-0002-6695-0944 \\ Faculdade São Leopoldo Mandic, Brasil \\ E-mail: diogoalves_15@yahoo.com.br \\ Victor da Mota Martins \\ ORCID: https://orcid.org/0000-0001-6631-6161 \\ Faculdade Patos de Minas, Brasil \\ E-mail: victortag@hotmail.com \\ Bruno Fontenele Carvalho \\ ORCID: https://orcid.org/0000-0002-9660-9958 \\ Faculdade São Leopoldo Mandic, Brasil \\ E-mail: bruno.carvalho.odt@gmail.com
}

\begin{abstract}
Resumo
A prevalência do câncer de cabeça e pescoço vem aumentando cada vez mais e está associado a dor, desfiguração, disfunção, sofrimento psicossocial e morte. Dentre as formas de tratamento existem a radioterapia, quimioterapia, cirurgia, assim como sua combinação. Uma das maiores complicações da radioterapia é a osteorradionecrose. Apresenta xerostomia, e consequentemente cáries por radiação, que aparecem devido a uma alteração na microbiota oral, criando um ambiente mais ácido. Esses micro-organismos causam uma inflamação no interior do dente que posteriormente evolui para uma necrose pulpar. Com isto, o objetivo deste trabalho é realizar uma revisão na literatura que evidencie como realizar um tratamento endodôntico atraumático, destacando a conduta para tratamento endodôntico em pacientes oncológicos e como evitar a osteoradionecrose dos maxilares. Foram utilizadas estratégias de busca em cinco bases de dados: PubMed; Cochrane; LILACS; Web of Science; e Scopus, com as palavras chave: "head neck cancer", "root canal therapy", "endodontic treatment". O principal motivo para realizar o tratamento endodôntico é o fato de conseguir-se realizar um tratamento mais conservador sendo uma alternativa para evitar a extração do dente e diminuir a possibilidade de causar osteonecrose. Em pacientes que estão na fase de pré tratamento radioterápico, o protocolo sugere a realização de um tratamento normal. Nas fases durante e pós tratamento por irradiação, a limpeza dos canais deve ser atraumática. Se um dente possuir estrutura suficiente, compatível com um bom prognóstico de reabilitação, porém com uma lesão inflamatória ou uma inflamação pulpar irreversível, o tratamento endodôntico é o ideal.
\end{abstract}

Palavras-chave: Endodontia; Neoplasias de cabeça e pescoço; Radioterapia.

\begin{abstract}
The prevalence of head and neck cancer has been increasing more and more and is associated with pain, disfigurement, dysfunction, psychosocial suffering and death. Among the forms of treatment there are radiotherapy, chemotherapy, surgery, as well as their combination. One of the biggest complications of radiotherapy is osteoradionecrosis. It presents xerostomia, and consequently radiation caries, which appear due to a change in the oral microbiota, creating a more acidic environment. These microorganisms cause an inflammation inside the tooth that later develops into pulp necrosis. With this, the objective of this work is to carry out a review in the literature that shows how to perform an atraumatic endodontic treatment, highlighting the conduct for endodontic treatment in cancer patients and how to avoid osteoradionecrosis of the jaws. Search strategies were used in five databases: PubMed; Cochrane; LILACS; Web of Science; and Scopus, with the keywords: "head neck cancer", "root canal therapy", "endodontic treatment". The main reason for performing endodontic treatment is the fact that a more conservative treatment can be performed, being an alternative to avoid tooth extraction and reduce the possibility of causing osteonecrosis. In patients who are in the pre-radiotherapy treatment phase, the protocol suggests a normal treatment. In the phases during and after irradiation treatment, the cleaning of the channels must be non-traumatic. If a tooth has sufficient structure, compatible with a good prognosis for rehabilitation, but with an inflammatory lesion or irreversible pulp inflammation, endodontic treatment is ideal.
\end{abstract}

Keywords: Endodontics; Head and neck neoplasms; Radiotherapy. 


\begin{abstract}
Resumen
La prevalencia del cáncer de cabeza y cuello aumenta cada vez más y se asocia con dolor, desfiguración, disfunción, sufrimiento psicosocial y muerte. Entre las formas de tratamiento se encuentran la radioterapia, la quimioterapia, la cirugía, así como su combinación. Una de las mayores complicaciones de la radioterapia es la osteorradionecrosis. Presenta xerostomía, y consecuentemente caries por radiación, que aparecen por un cambio en la microbiota oral, creando un ambiente más ácido. Estos microorganismos provocan una inflamación dentro del diente que luego se convierte en necrosis pulpar. Con ello, el objetivo de este trabajo es realizar una revisión en la literatura que muestre cómo realizar un tratamiento endodóntico atraumático, destacando la conducta para el tratamiento endodóntico en pacientes con cáncer y cómo evitar la osteorradionecrosis de los maxilares. Se utilizaron estrategias de búsqueda en cinco bases de datos: PubMed; Cochrane; LILACS; Web de la Ciencia; y Scopus, con las palabras clave: "cáncer de cabeza y cuello", "terapia de conducto", "tratamiento de endodoncia". El principal motivo para realizar un tratamiento endodóntico es el hecho de que se puede realizar un tratamiento más conservador, siendo una alternativa para evitar la extracción dentaria y reducir la posibilidad de provocar osteonecrosis. En pacientes que se encuentran en la fase de tratamiento previo a la radioterapia, el protocolo sugiere un tratamiento normal. En las fases durante y después del tratamiento de irradiación, la limpieza de los canales debe ser no traumática. Si un diente tiene estructura suficiente, compatible con un buen pronóstico para la rehabilitación, pero con una lesión inflamatoria o inflamación pulpar irreversible, el tratamiento endodóntico es ideal.
\end{abstract}

Palabras clave: Endodoncia; Neoplasias de cabeza y cuello; Radioterapia.

\title{
1. Introdução
}

A prevalência do câncer de cabeça e pescoço vem aumentando cada vez mais e está associado a dor, desfiguração, disfunção, sofrimento psicossocial e morte (Chow, 2020). Dentre as suas formas de tratamento existem a radioterapia, quimioterapia, cirurgia, assim como a combinação de algumas ou até mesmo todas elas (Lieshout \& Bots, 2014). As opções terapêuticas variam de acordo com fatores epidemiológicos, localização anatômica e estágio (Chow, 2020). O maior desafio da irradiação da região de cabeça e pescoço, se deve ao grande número de estruturas vitais na região. Esse tipo de tratamento pode causar vários efeitos secundários indesejáveis, sendo um deles a destruição de estruturas orais, ocasionando uma diminuição na qualidade de vida do paciente (Lieshout \& Bots, 2014).

Desde 1976, um autor já mencionava as três formas de terapia por irradiação para tratamento de câncer (Cox,1976). Como tratamento pré-cirúrgico, para diminuição e tentativa de eliminar extensões da lesão, fazendo com que houvesse menor risco de recidiva. Como tratamento pós-cirúrgico, para eliminar lesões residuais. E terceiro, como terapia única, sem necessidade de intervenção cirúrgica. A eficácia da radioterapia existe por causa da radiação ionizante, que inibe e controla o crescimento celular. No entanto, para atingir as células alvo, essa radiação passa antes por células sadias, alterando e danificando o DNA delas. Por isso, várias técnicas estão sendo desenvolvidas para minimizar estes efeitos destrutivos, dentre elas, a radioterapia fracionada alternada (Kataoka et al., 2012).

A terapia por irradiação envolve o uso de uma dose de radiação correta e controlada, direcionada à massa tumoral. A variável utilizada para a dose de radiação absorvida é "gray" (Gy). Os efeitos colaterais da irradiação da região de cabeça e pescoço são bem conhecidos, sendo muito relacionados a doses, sendo fracionados durante a semana, por um período de 5 a 7 semanas (Bodrumlu et al, 2009). Juntamente com a cirurgia, a radioterapia representa uma das principais opções de tratamento com intenção curativa, tanto em estágios iniciais quanto avançados, com doses variando de 54 a 70 Gy, administradas com um esquema de fracionamento padrão de 2 Gy / fração, uma fração / dia, 5 frações / semana. Além disso, em ambientes de alto risco, a combinação de radioterapia com cisplatina concomitante (100 mg / m2 a cada 3 semanas) é a abordagem não cirúrgica preconizada (Alterio et al.,2019).

Quase todos os pacientes com câncer avançado de cabeça e pescoço sofrem complicações do tratamento com radioterapia ou quimiorradioterapia (Epstein et al., 2012), que é atualmente o padrão de atendimento com ou sem cirurgia para câncer em estágio avançado. Há um aumento da frequência e gravidade dos efeitos colaterais, particularmente quando a quimioterapia é combinada com regimes de radioterapia acelerado ou hiperfracionado (Zecha et al., 2016).

Uma das maiores e mais sérias complicações da radioterapia é a osteorradionecrose, sendo ela uma necrose isquêmica 
do osso. Esta condição não possui uma previsibilidade notável, podendo ela ser superficial ou profunda, de evolução lenta ou rápida. A vascularização sofre uma alteração de sua atividade como um todo após a irradiação naquela região. Essas alterações incluem a diminuição de transporte de oxigênio para as células, assim como uma menor chegada de nutrientes e células de defesa. No entanto não existem estudos que comprovem quando ocorre a evolução desta complicação, sugerindo ao paciente e aos profissionais da saúde, que estejam sempre atentos aos sinais e sintomas da doença. Estes sinais e sintomas incluem fístulas intrabucal ou extrabucal, trismo, dor, dificuldades mastigatórias, fratura patológica, infecção local e drenagem de secreção purulenta (Grimaldi et al., 2005).

Os efeitos colaterais desse tipo de tratamento têm sido amplamente abordados na literatura. Os principais efeitos orais são danos causados às glândulas salivares e aos ossos maxilar e principalmente mandibular. Um dos efeitos mais relatados por pacientes é a xerostomia, por ter grande impacto na qualidade de vida. Em associação com a xerostomia, temos também as cáries por radiação, que começam a aparecer devido a uma alteração repentina na microbiota oral, criando um ambiente mais ácido. Se não tratadas imediatamente, essas lesões de cárie podem rapidamente evoluir, criando uma cavidade tão profunda que gera o acesso de micro-organismos externos para o espaço pulpar do dente. Esses micro-organismos causam uma inflamação no interior do dente que posteriormente evolui para uma necrose pulpar. Esta necrose pulpar eventualmente ocasiona o desenvolvimento de uma lesão inflamatória periapical, doença que requer o tratamento endodôntico ou exodontia para eliminação deste foco de infecção (Hommez et al., 2012). O aumento do número de lesões por cáries, também estão relacionados à dieta do paciente que, durante ou após a radioterapia, passa a ser uma dieta líquida e pastosa rica em carboidratos para aumentar ou manter o peso (Lieshout \& Bots, 2014).

Uma revisão narrativa foi publicada buscando evidenciar como a saliva pode ser uma das principais vias de transmissão dos vírus em lesões periapicais assim como a omissão do isolamento absoluto podem facilitar de entrada de alguns microorganismos durante o tratamento endodôntico, no entanto, não foram encontrados resultados referentes a presença de microorganismos nos tecidos periapicais mas estes podem ser associados a capacidade de exacerbar a resposta inflamatória, potencializar a reabsorção óssea e prejudicar na reparação tecidual periapicail (Loureiro et al., 2021)

Com isto, o objetivo deste trabalho é realizar uma revisão na literatura que evidencie como realizar um tratamento endodôntico atraumático, destacando a conduta para tratamento endodôntico em pacientes oncológicos e como evitar a osteoradionecrose dos maxilares.

\section{Metodologia}

A pesquisa bibliográfica foi realizada em abril de 2021. As bases de dados, Literatura Latino-Americana e do Caribe em Ciências da Saúde (LILACS), PubMed (incluindo MedLine), SciELO, Scopus e Web of Science foram as fontes primária de estudo. De forma complementar, uma busca foi realizada nas referências dos estudos potencialmente elegíveis.

Os recursos MeSH (Medical Subject Headings) e DeCS (Health Sciences Descriptors) foram utilizados para selecionar os descritores de pesquisa. Os operadores booleanos "AND" e "OR" aprimoraram a estratégia de pesquisa por meio de várias combinações. Foram utilizadas as palavras chave: "head neck cancer", "root canal therapy", "endodontic treatment" sem limitação de tempo. Dentro dos critérios de inclusão estavam pacientes que receberam tratamento endodôntico, antes, durante ou após tratamento radioterápico independentemente da idade; estudos laboratoriais, in vitro, in vivo, in situ, transversais, longitudinais e revisões sistemáticas de literatura Foram considerados elegíveis estudos que apresentassem pelo menos um dos seguintes desfechos: (1) osteonecrose dos maxilares; (2) sucesso do tratamento endodôntico. Não foram aplicadas restrições. 


\section{Revisão de Literatura}

\subsection{Osteorradionecrose}

Em um trabalho, os autores discutem as prováveis causas da osteorradionecrose e o tratamento mais indicado para quando alguém é diagnosticado com essa complicação e mencionam que os traumas gerados por extrações e próteses dentárias são sim fatores que influenciam no desenvolvimento da osteorradionecrose, e baseiam suas conclusões em alguns estudos. Se destaca dentre estes estudos, um, que analisa oitenta pacientes com complicações sistêmicas, e o mesmo estudo indica que metade deste número é referente a pacientes que passaram por procedimentos de exodontia durante ou após o tratamento com irradiação (Grimaldi et al., 2005).

A osteonecrose porém, não ocorre somente em pacientes que estão sendo submetidos à radioterapia. Existem outros tratamentos/medicamentos que podem causá-la, dentre eles, os bisfosfonatos. Os bisfosfonatos são as principais drogas utilizadas para preservação da estrutura óssea, quando necessária. Algumas das condições e situações de emprego para elas são em casos de câncer metastático, osteoporose, doença de Paget e artrite reumatoide. Os principais eventos que desencadeiam a osteonecrose são extrações dentárias e próteses mal-adaptadas e a terapia de canal radicular pode adiar ou mesmo erradicar a necessidade de extrações dentárias de dentes cariados em pacientes que fazem o uso de bifsosfonatos (Kyrgidis et al., 2010). A Associação Americana de Cirurgiões Orais e Maxilofaciais (AAOMS) publicou em 2009, um guia que preconiza o tratamento endodôntico para dentes com prognóstico desfavorável para ser restaurado em paciente sob tratamento com bisfosfonatos, para assim minimizar os riscos de osteonecrose (Ruggiero et al., 2014).

É consenso que o tratamento mais indicado inicialmente para a osteorradionecrose deve ser o mais conservador possível. Este tratamento inicial consiste em debridamento, limpeza e antibioticoterapia, podendo ou não ser acompanhado de cirurgias de pequeno porte. Em casos que não apresentam resposta satisfatória ao tratamento conservador, utiliza-se técnica de oxigenação hiperbárica. Neste tipo de tratamento, ocorre a neovascularização, aumento do número de células e aumento da atividade celular, indicando um meio adequado para a cicatrização dos tecidos afetados pela radiação. Já em casos muito persistentes, o mais indicado são as cirurgias mais invasivas, que envolvem uma extensa remoção da região envolvida pela osteorradionecrose, necessitando em seguida em vários casos de uma reconstrução também (Grimaldi et al., 2005).

\subsection{Osteonecrose induzida por bisfosfonatos}

Os bisfosfonatos são essenciais no tratamento da osteoporose e de certos tipos de câncer. Os Estados Unidos aprovaram o primeiro medicamento da classe para o tratamento da osteoporose em 1995. Os bisfosfonatos de primeira geração que não contem nitrogênio induzem apoptose pela formação de um análogo tóxico de trifosfato de adenosina, enquanto a segunda e terceira gerações, contendo nitrogênio, inibem a função dos osteoclastos ao direcionar a enzima farnesil difosfato sintase, que é necessária para modificação pós-tradução de pequenas proteínas de ligação a trifosfato de guanosina (AlRahabi \& Ghabbani 2018).

Os bisfosfonatos são análogos do pirofosfato substituídos por carbono que são inibidores potentes da reabsorção óssea mediada por osteoclastos. Esses compostos têm especificidade para o osso devido à sua alta afinidade de ligação para os fosfatos de cálcio. Essas drogas não são bem metabolizadas e são liberadas lentamente por longos períodos de tempo. As últimas gerações dessas drogas incluem alendronato (Fosamax, Merck, Whitehorse Station, NJ), risedronato (Actonel, Aventis, Bridgewater, NJ), pamidronato (Aredia, Novartis, East Hanover, NJ) e zoledronato (Zometa, Novartis, Leste Hanover, NJ). Seu modo de ação ainda não está claro, mas são conhecidos por inibir a função osteoclástica, induzir apoptose de osteoclastos e inibir a diferenciação de osteoclastos de precursores e seu mecanismo de ação para alterar a angiogênese também não é claro e pode ser variável (Sarathy, Bourgeois \& Goodell 2005).

Bisfosfonatos induzem apoptose de osteoclastos, como resultado, a formação óssea supera a reabsorção óssea devido 
o desequilíbrio de osteoblastos e osteoclastos na superfície óssea. Os antirreabsortivos são drogas úteis para o tratamento da osteoporose e do câncer, no entanto, em 2003, a osteonecrose da mandíbula relacionada com bisfosfonatos (BRONJ) foi relatada em pacientes oncológicos que receberam bisfosfonatos intravenosos (Kuroshima, Sasaki \& Sawase 2019). O termo relacionado à medicação (MRONJ) foi proposto pela American Association of Oral and Maxillofacial Surgeons (AAOMS) em 2014 em vez de BRONJ e/ou DRONJ já definidos em 2009 (Ruggiero et al., 2014).

É importante salientar que o uso de bisfosfonatos também podem ser uma das causas da osteonecrose em tratamentos traumáticos.

\subsection{Tratamento endodôntico atraumático}

O principal motivo para optar-se pela realização do tratamento endodôntico no lugar de um tratamento mais invasivo é o fato de conseguir-se realizar um tratamento endodôntico mais conservador. O tratamento do canal radicular serve como uma alternativa de tratamento para a extração do dente e diminui a possibilidade dessa modalidade de tratamento causar osteonecrose da mandíbula relacionada ao bisfosfonato (Kaptan, Kazandag \& Iseri 2013).

Para que esse tratamento endodôntico seja o mais conservador possível, é necessário realizar um tratamento atraumático. $\mathrm{O}$ tratamento atraumático precisa ter menos injúrias e desgastes na região óssea perapical para que não haja riscos do desenvolvimento de uma osteonecrose. Este tipo de tratamento também precisa proporcionar mais velocidade e menos desconforto, para que não cause muito desgaste ao paciente. Para que o tratamento seja atraumático, é preciso estabelecer algumas regras e utilizar equipamentos adequados. A primeira regra é trabalhar sempre no comprimento real do dente, não o ultrapassando para não o tornar um procedimento invasivo (Ferraz et al., 2004).

O segundo passo é a utilização de equipamentos para deixar o tratamento mais rápido e eficaz. Dentre os equipamentos disponíveis no dia-a-dia, independentemente do local de realização do tratamento, existem os localizadores foraminais, que oferecem maior precisão e agilidade na aquisição do comprimento real do dente, além dos instrumentos rotatórios, que, se empregados da forma correta, oferecem maior eficácia na limpeza mecânica dos canais e maior agilidade também, além de tecnologias para anestesia, radiografias digitais, tomografia computadorizada de feixe cônico, unidades de ultrassom, lasers, microscópios operacionais, conceitos 3D de obturação e selantes 100\% inorgânicos (Camargo et al., 2021). É importante salientar que o número de sessões também é um fator de grande influência para que o tratamento seja atraumático, devendo ser sempre que possível em sessão única para dentes com polpa vital, e em mais de uma sessão, quando possível, para dentes com polpa necrosada (Ferraz et al., 2004; AlRahabi \& Ghabbani, 2018).

Por último, é importante atentar-se à solução irrigadora que será empregada durante o tratamento. Para que não haja nenhum estímulo ao desenvolvimento de uma reação inflamatória, é recomendado evitar soluções cáusticas, como o hipoclorito de sódio. Para substituir esta solução irrigadora, deve ser empregado o uso de clorexidina para dentes já necrosados (Ferraz et al., 2004).

Alternativas adequadas podem incluir tratamento de canal radicular não cirúrgico se a doença pulpar for identificada. Dois casos clínicos ilustram tratamentos pulpares e perirradiculares precisos, resultando em diagnósticos claros, antes de prosseguir com o tratamento endodôntico. Deve-se tomar cuidado na colocação de grampos e lençóis de borracha para evitar lesões na mucosa que podem precipitar a inflamação e a doença. O tratamento endodôntico cirúrgico não é recomendado e deve ser considerado contra-indicado em pacientes em uso de pamidronato ou zoledronato (Sarathy, Bourgeois\& Goodell, 2005).

Um estudo recente avaliou a influência das lesões periapicais na potencialização da osteonecrose após a extração do dente e mostrou que houve o aumento da ocorrência de osteonecrose relacionada ao medicamento, destacando que as lesões periapicais devem ser controladas antes da extração dentária, para minimizar o risco de necrose dos maxilares (AlRahabi \& 
Ghabbani, 2018).

\section{Discussão}

Para evidenciar a conduta para tratamento endodôntico em pacientes oncológicos, é necessário atenta-se a algums fatores. A radioterapia realizada antes do tratamento endodôntico reduz a resistência de união do material obturador à dentina radicular, independente do tipo de cimento utilizado, uma vez que danifica a rede de fibras colágenas da dentina (Martins et al., 2016; Paiola, 2018).

A comunicação com o médico do paciente são as primeiras e mais essenciais etapas antes de qualquer procedimento. O enxágue bucal com clorexidina por um minuto, antes de iniciar o tratamento, reduz o número de microrganismos na cavidade oral e minimiza a possibilidade de bacteremia que pode estar presente em decorrência de trauma em tecidos moles. Evitar o uso de agentes anestésicos com vasoconstritores para reduzir o risco de vascularização inadequada, pois os bisfosfonatos possuem efeitos antiangiogênicos. Minimizar o trauma, principalmente nos tecidos moles, durante o isolamento absoluto com grampos e dique de borracha. Executar o trabalho em um ambiente estritamente asséptico. Desinfetar o dente e o dique de borracha esfregando-os com uma solução desinfetante adequada por 2 minutos. Evitar a permeabilidade do forame apical, o que pode aumentar a possibilidade de bacteremia por extrusão de detritos, neste caso, localizadores de ápice eletrônicos são usados para determinar a constrição apical. Durante a instrumentação do canal radicular, usar sistemas rotativos de níquel titânio (NiTi) e evitar sistemas alternativos, que podem causar mais extrusão de detritos. Aplicar técnicas de obturação com riscos mínimos de obturação e extensão excessiva, para aumentar a eficiência do tratamento do canal radicular e reduzir a possibilidade de irritação do tecido periapical. A técnica de compactação lateral fria minimiza o risco de preenchimento excessivo do canal radicular em comparação com a técnica de aquecimento. Considerar como pacientes de alto risco aqueles em terapia por mais de 3 anos e pacientes com problemas sistêmicos, como doença renal crônica, diabetes e corticoterapia, pois requerem cuidados especiais. O uso profilático de antibióticos em pacientes tratados com bisfosfonato antes de um canal radicular não cirúrgico não alcançou consenso, recomendando então antibióticos profiláticos nos seguintes casos: polpas necróticas em pacientes submetidos ao tratamento com bisfosfonatos, pacientes tratados com há mais de 3 anos e pacientes com vários dentes que requerem tratamento de canal radicular. Programar todos os tratamentos em uma única consulta, para limitar a dosagem da profilaxia antibiótica (AlRahabi \& Ghabbani, 2018).

Assim para padronizarmos os tratamentos endodônticos em pacientes oncológicos, seria interessante seguir uma conduta específica para cada situação. Em pacientes que estão na fase de pré tratamento radioterápico, o protocolo sugere a realização de um tratamento normal, contanto que o paciente tenha um espaço de duas semanas entre a última sessão do tratamento endodôntico e o início da irradiação de cabeça e pescoço. Nas fases durante e pós tratamento por irradiação, a limpeza dos canais deve ser atraumática, como citada anteriormente, evitando a penetração de materiais nos tecidos periapicais. A obturação também deve respeitar esse limite de comprimento real do dente, pois uma sobre obturação também pode desencadear uma reação inflamatória (Rodrigues et al., 2006).

Em contrapartida ao tratamento endodôntico, temos o tratamento à base de exodontias. É de suma importância sempre aplicar a técnica mais indicada para cada caso. Quando o tratamento endodôntico é comparado à exodontia, ambos possuem vantagens e desvantagens, assim como indicações e contraindicações. A endodontia é deixada de lado, quando o dente em questão está muito fragilizado e possui prognóstico ruim, se não for extraído. Contudo só pode ser feita a exodontia, se o paciente tem possibilidade de aguardar um prazo mínimo de 2 semanas da data da exodontia até o início do tratamento oncológico com irradiação de cabeça e pescoço. Caso contrário, as chances de desenvolvimento de uma osteorradionecrose são consideráveis (Rosales et al., 2009). Assim sendo, devem ser evitadas ao máximo as exodontias após o início do tratamento de irradiação de cabeça e pescoço. 
As principais regiões anatômicas acometidas pelo câncer de cabeça e pescoço são: a cavidade oral que inclui os lábios, mucosa bucal, língua anterior, assoalho da boca, palato duro, gengiva e trígono retromolar. A faringe, que inclui a nasofaringe situada atrás da cavidade nasal, orofaringe, compreendendo a área tonsilar, base da língua, palato mole e parede posterior da faringe e hipofaringe, compreendendo os seios piriformes, superfície posterior da laringe e área pós-cricoide, e paredes faríngeas inferior posterior e ínfero-lateral. A laringe inclui a laringe supraglótica, laringe glótica, cordas vocais verdadeiras e comissuras anterior e posterior, e laringe subglótica. A cavidade nasal e os seios paranasais incluem os seios maxilar, etmóide, esfenoidal e frontal. A inserção mostra as características histológicas típicas do carcinoma de células escamosas que podem ser vistas no câncer de cabeça e pescoço (Chow, 2020).

Juntamente com a cirurgia, a radioterapia representa uma das principais opções de tratamento com intenção curativa, tanto em estágios iniciais quanto avançados, com doses administradas com um esquema de fracionamento padrão e a combinação com medicamentos (Alterio et al., 2019).

Terapias administradas separadamente e combinadas podem gerar sintomas adicionais como complicações agudas que incluem mucosite oral, dor, disfagia, infecções, alterações salivares, disgeusia e dermatite. Complicações crônicas comuns incluem hipossalivação e xerostomia, infecções da mucosa, atrofia da mucosa, neuropatias incluindo dor da mucosa, disgeusia, desmineralização dentária e cárie rampante, progressão da periodontite, necrose óssea, mucocutânea e fibrosis, disfagia, trismo, linfedema, dermatite e alterações da voz e da fala (Vissink et al., 2003; Zecha et al., 2016).

Em diversas partes do tratamento por irradiação de cabeça e pescoço deve-se avaliar o procedimento mais indicado para cada situação, com o objetivo de minimizar o risco de desenvolvimento de uma osteorradionecrose. Não pode ser aplicado um tratamento que envolva exodontias para solucionar alguma complicação que possa ser resolvida com um tratamento endodôntico primeiro. Porém também não é recomendado que o paciente fique sendo exposto a um tratamento endodôntico que não tem um bom prognóstico.

A literatura fornece evidências de seus efeitos colaterais diretos na estrutura dentária, incluindo alterações nas propriedades mecânicas e na composição química do esmalte e da dentina, a radiação gera diferentes efeitos nas bandas de fosfato e carbonato. A menor cristalinidade de carbonato nos dentes irradiados pode ser explicada por um maior número de íns carbonato planos substituindo os íons fosfato tetraédricos na estrutura da hidroxiapatita, aumentando a desordem da estrutura cristalina. Como o colágeno é a proteína mais abundante da dentina (90\%), sua proteólise tem impacto mais significativo na integridade estrutural desse tecido, o que pode ser facilmente observado em elementos dentários afetados por lesões de cárie que ficam comprometidas mecânica e funcionalmente. $\mathrm{O}$ aumento do risco de cárie associada à radiação em pacientes submetidos à radioterapia de cabeça e pescoço se deve não apenas a alterações salivares, dietéticas e microbiológicas, mas também a alterações na composição química da estrutura dentária (de Miranda et al., 2018)

Ocorrem também outras alterações de grande importância em pacientes que foram irradiados na região de cabeça e pescoço. Uma análise sugere que existe uma alteração significativa não somente na microbiota oral, mas também na microbiota intra-pulpar e intra-canal durante a radioterapia. Essas alterações podem de fato contribuir para um desequilíbrio dessas populações, facilitando o desenvolvimento de uma reação inflamatória endodôntica (Hommez, 2012).

A vitalidade pulpar preservada impacta positivamente na longevidade do dente restaurado, porém a alta incidência de cáries, traumas dentários e intervenções iatrogênicas podem levar a processos degenerativos da polpa. Alterações nas propriedades mecânicas da dentina causadas pela ação de agentes de irrigação, medicamentos e materiais obturadores de canais radiculares podem predispor à fratura dentária. Além disso, a perda da integridade estrutural pode aumentar a ocorrência de fratura. Pacientes com tumores de cabeça e pescoço sob tratamento radioterápico podem apresentar alguns distúrbios orais e a complicação mais evidente para a dentição é a cárie induzida por radiação, no entanto, o efeito adicional causado pela terapia endodôntica realizada em dentes irradiados permanecia desconhecido. Pacientes com dentes obturados que requerem 
radioterapia podem ter mais alterações nas propriedades mecânicas do substrato da dentina radicular (Novais et al., 2016)

Em um estudo de Kataoka, S. H. et al em 2012, pacientes foram avaliados em diversas fases durante um tratamento de irradiação na região de orofaringe. Estes foram submetidos ao teste de sensibilidade pulpar ao frio no início e no final do tratamento, sendo que todos responderam ao teste de forma positiva no início do tratamento. Já no final, após terem sido submetidos a altas doses de irradiação, nenhuma paciente estava com respostas positivas ao teste de sensibilidade pulpar. Este estudo serve de grande exemplo, demonstrando que todos os testes, exames e informações devem ser levados em consideração, necessitando de uma avaliação multidisciplinar para se obter um correto plano de tratamento.

Já em outro trabalho, é possível afirmar que a radioterapia foi capaz de alterar a composição química da dentina humana. A radiação pode influenciar a troca de íons fosfato-carbonato na hidroxiapatita e aumentar ainda mais a concentração dos componentes orgânicos na dentina. Além disso, a dentina coronária é mais mineralizada que a radicular (de Miranda et al., 2018). Quando um paciente chega no consultório, um dos testes mais empregados para saber o diagnóstico pulpar dele é o teste de sensibilidade pulpar com estímulo frio. O único problema é a incerteza se estes testes estão sendo aplicados de forma correta, pois eles são grandes auxiliares para definir um plano de tratamento correto.

Além dos testes de sensibilidade pulpar, temos também os testes de percussão e os exames complementares, que englobam os exames radiográficos. O teste de sensibilidade pulpar dirá basicamente se existe uma pulpite, em que fase esta pulpite está, ou se o dente está necrosado. Dependendo do resultado, a conduta pode ser um tratamento simples restaurador, um tratamento endodôntico, ou a exodontia do dente. Ou seja, pode-se dizer que se o teste é realizado de forma equivocada, o plano de tratamento também tem grandes chances de estar errado, fazendo com que o paciente passe por um procedimento de exodontia ou de tratamento endodôntico sem necessidade.

A polpa é constituída por várias estruturas, cada uma delas exercendo um papel fundamental. Uma dessas atuações essenciais dela é a sensibilidade gerada em casos de traumas, lesões por cárie, ou qualquer outra alteração. Essa sensibilidade serve como feedback, para que se possa reagir e tomar providências contra estes estímulos externos. Uma nova hipótese que justifica a frequente formação de cáries em pacientes que foram irradiados, relata que ocorre uma alteração no metabolismo dos odontoblastos e diminuição da resposta do complexo dentino-pulpar, diminuindo ou inibindo a sensibilidade a dor em até mesmo lesões de cárie muito profundas. Ocorre também a diminuição da vascularização e inervação, fazendo com que a saturação de oxigênio fique escassa (Faria et al., 2014). Essa informação é de grande relevância às cáries de radiação, pois explica o grande número de pacientes que possuem lesões de cáries muito profundas após a radioterapia. Testes de sensibilidade pulpar foram realizados em 40 pacientes tratados com radiação. Após uma radiação de 60Gy a 70Gy, nenhum desses pacientes responderam de forma positiva a este teste, confirmando que realmente ocorrem alterações na polpa (Kataoka et al., 2012).

As células inflamatórias ativadas secretam múltiplas proteínas, incluindo citocinas (ou seja, interleucina e proteínas degradantes como colagenase ou proteinases, que interferem na cicatrização. As endotoxinas bacterianas irão ativar o sistema complemento, todos esses fatores provocam a diferenciação de macrófagos em osteoclastos (AlRahabi \& Ghabbani, 2018). Em 2006, Rodrigues, H. M. et al já dizia que em um caso de abscesso periapical, resultante de uma lesão de cárie durante o tratamento por irradiação de cabeça e pescoço, o mais indicado, caso seja possível realizar a restauração ou reabilitação do dente posteriormente, é o tratamento endodôntico. Os abscessos são precipitadores de osteorradionecrose e devem ser tratados imediatamente após o diagnóstico. Lembrando que um tratamento endodôntico em paciente sob tratamento oncológico, deve ser sempre o mais conservador possível, evitando extravasamento de soluções cáusticas e processos traumáticos além do comprimento real do dente, pois os bisfosfonatos têm como alvo a reabsorção óssea mediada por osteoclastos e inibem a remodelação óssea. Esse processo pode interferir na cicatrização de lesões periapicais após tratamento de canal radicular e podem ser consideradas fatores predisponentes a agravar o desenvolvimento de necrose dos maxilares. 
Melhor que um plano de tratamento correto, seria um plano de prevenção correto. Kyrgidis, A. et al já mencionava em seu trabalho em 2010, que a um paciente ao realizar qualquer tipo de tratamento oncológico, envolvendo radiação ou não, deve ser oferecido sempre um tratamento preventivo de qualidade, visando assim diminuir as chances de este mesmo paciente precisar de um tratamento endodôntico ou de extração dentária.

Oncologistas e dentistas devem ser amplamente alertados sobre essa possível complicação, para que pacientes em uso de bisfosfonatos e considerando procedimentos odontológicos eletivos possam ser devidamente orientados. Um exame dentário completo e as exodontias necessárias com tempo para a cura são recomendados antes de iniciar a terapia com bisfosfonatos (Sarathy, Bourgeois\& Goodell, 2005).

\section{Considerações Finais}

Ficou claro que o mais importante e almejado nos tempos de hoje é prevenir e promover o conservadorismo. Após um bom plano de tratamento e avaliação de cada caso, deve-se sempre optar por reabilitar ou restaurar um dente sem grandes desgastes e procedimentos invasivos. Se um dente possuir uma forte estrutura, compatível com um bom prognóstico de reabilitação, porém com uma lesão inflamatória ou uma inflamação pulpar irreversível, opta-se pelo tratamento endodôntico. Somente em casos onde nenhum dos tratamentos restauradores e reabilitadores são indicados, é que se escolhe a extração do dente. Portanto deve-se sempre, independente da fase do tratamento de irradiação de cabeça e pescoço, realizar um planejamento e tratamento multidisciplinar, a fim de otimizar a qualidade de vida do paciente tanto no tratamento odontológico, como no tratamento oncológico.

\section{Referências}

AlRahabi, M. K., \& Ghabbani, H. M. (2018). Clinical impact of bisphosphonates in root canal therapy. Saudi medical journal, 39(3), 232-238. https://doi.org/10.15537/smj.2018.3.20923

Alterio, D., Marvaso, G., Ferrari, A., Volpe, S., Orecchia, R., \& Jereczek-Fossa, B. A. (2019). Modern radiotherapy for head and neck cancer. Seminars in oncology, 46(3), 233-245. https://doi.org/10.1053/j.seminoncol.2019.07.002

Baumgartner, J. C., \& Eggli, D. (1989). The use of bone imaging to detect a periapical lesion of endodontic origin. Journal of endodontics, 15(10), 493-495. https://doi.org/10.1016/s0099-2399(89)80032-9

Bodrumlu, E., Avsar, A., Meydan, A. D., \& Tuloglu, N. (2009). Can radiotherapy affect the apical sealing ability of resin-based root canal sealers? Journal of the American Dental Association (1939), 140(3), 326-330. https://doi.org/10.14219/jada.archive.2009.0162

Brennan, M. T., Woo, S. B., \& Lockhart, P. B. (2008). Dental treatment planning and management in the patient who has cancer. Dental clinics of North America, 52(1), 19-vii. https://doi.org/10.1016/j.cden.2007.10.003

Camargo, J. M. P. De.; Camargo, R. V. De.; Cintra, L. T. A.; Faria, F. D.; Consolaro, A.; Cao, D.; Leonardo, R. De T. (2021). Salto Evolutivo em Endodontia. Relato de caso. Research, Society and Development, 10(3), e44110313538 DOI: 10.33448/rsd-v10i3.13538.

Cox F. L. (1976). Endodontics and the irradiated patient. Oral surgery, oral medicine, and oral pathology, 42(5), 679-684. https://oi.org/10.1016/00304220(76)90218-8

Chow L. (2020). Head and Neck Cancer. The New England journal of medicine, 382(1), 60-72. https://doi.org/10.1056/NEJMra1715715

de Miranda, R. R., Silva, A., Dantas, N. O., Soares, C. J., \& Novais, V. R. (2019). Chemical analysis of in vivo-irradiated dentine of head and neck cancer patients by ATR-FTIR and Raman spectroscopy. Clinical oral investigations, 23(8), 3351-3358. https://doi.org/10.1007/s00784-018-2758-6

Epstein, J. B., Thariat, J., Bensadoun, R. J., Barasch, A., Murphy, B. A., Kolnick, L., Popplewell, L., \& Maghami, E. (2012). Oral complications of cancer and cancer therapy: from cancer treatment to survivorship. CA: a cancer journal for clinicians, 62(6), 400-422. https://doi.org/10.3322/caac.21157

Faria, K. M., Brandão, T. B., Ribeiro, A. C., Vasconcellos, A. F., de Carvalho, I. T., de Arruda, F. F., Castro Junior, G., Gross, V. C., Almeida, O. P., Lopes, M. A., \& Santos-Silva, A. R. (2014). Micromorphology of the dental pulp is highly preserved in cancer patients who underwent head and neck radiotherapy. Journal of endodontics, 40(10), 1553-1559. https://doi.org/10.1016/j.joen.2014.07.006

Ferraz, F. C., Simões, W., Rapaport, A., \& Bozzo, R. O. (2004). O Uso de Localizador Apical Endodôntico em Pacientes Irradiados. Revista Gaúcha de Odontologia, 52 (3), 157-160.

Grimaldi, N., Sarmento. V., Provedel, L ., \& Almeida, D., Cunha, S.(2005). Conduta do cirurgião-dentista na prevenção e tratamento da osteorradionecrose: revisão de literatura. Revista Brasileira de Cancerologia, 51(4), 319-324. 
Hommez, G. M., De Meerleer, G. O., De Neve, W. J., \& De Moor, R. J. (2012). Effect of radiation dose on the prevalence of apical periodontitis--a dosimetric analysis. Clinical oral investigations, 16(6), 1543-1547. https://doi.org/10.1007/s00784-011-0665-1

Kaptan, F., Kazandag, M. K., \& Iseri, U. (2013). Treatment of bisphosphonate related osteonecrosis following root canal therapy at the 1-year follow-up: report of two cases. Therapeutics and clinical risk management, 9, 477-482. https://doi.org/10.2147/TCRM.S52630

Kataoka, S. H., Setzer, F. C., Fregnani, E. R., Pessoa, O. F., Gondim, E., Jr, \& Caldeira, C. L. (2012). Effects of 3-dimensional conformal or intensitymodulated radiotherapy on dental pulp sensitivity during and after the treatment of oral or oropharyngeal malignancies. Journal of endodontics, 38(2), 148152. https://doi.org/10.1016/j.joen.2011.09.022

Kuroshima, S., Sasaki, M., \& Sawase, T. (2019). Medication-related osteonecrosis of the jaw: A literature review. Journal of oral biosciences, 61(2), 99-104. https://doi.org/10.1016/j.job.2019.03.005

Kyrgidis, A., Arora, A., Lyroudia, K., \& Antoniades, K. (2010). Root canal therapy for the prevention of osteonecrosis of the jaws: an evidence-based clinical update. Australian endodontic journal: the journal of the Australian Society of Endodontology Inc, 36(3), 130-133. https://doi.org/10.1111/j.17474477.2010.00280.x

Lieshout, H. F., \& Bots, C. P. (2014). The effect of radiotherapy on dental hard tissue--a systematic review. Clinical oral investigations, 18(1), 17-24. https://doi.org/10.1007/s00784-013-1034-z

Loureiro, C.; Seron, M. A.; Braga, G. P. De A.; Silva, C. C. Da; Jacinto, R. C.; Sivieri-Araujo, G.; Duque, C.; Cintra, L. T. A.; Gomes-Filho, J. E. (2021). Influência da infecção viral no processo de reparo das lesões periapicais: uma revisão narrativa. Research, Society and Development, 10(3), e14210313134, $10.33448 /$ rsd-v10i3.13134.

Martins, C. V., Leoni, G. B., Oliveira, H. F., Arid, J., Queiroz, A. M., Silva, L. A., \& Sousa-Neto, M. D. (2016). Influence of therapeutic cancer radiation on the bond strength of an epoxy- or an MTA-based sealer to root dentine. International endodontic journal, 49(11), 1065-1072. https://doi.org/10.1111/iej.12556

Novais, V. R., Soares, P. B., Guimarães, C. M., Schliebe, L. R., Braga, S. S., \& Soares, C. J. (2016). Effect of Gamma Radiation and Endodontic Treatment on Mechanical Properties of Human and Bovine Root Dentin. Brazilian dental journal, 27(6), 670-674. https://doi.org/10.1590/0103-6440201601267

Paiola, F. G., Lopes, F. C., Mazzi-Chaves, J. F., Pereira, R. D., Oliveira, H. F., Queiroz, A. M., \& Sousa-Neto, M. D. (2018). How to improve root canal filling in teeth subjected to radiation therapy for cancer. Brazilian oral research, 32, e121. https://doi.org/10.1590/1807-3107bor-2018.vol32.0121

Rosales, A. C., Esteves, S. C., Jorge, J., Almeida, O. P., \& Lopes, M. A. (2009). Dental needs in Brazilian patients subjected to head and neck radiotherapy. Brazilian dental journal, 20(1), 74-77. https://doi.org/10.1590/s0103-64402009000100013

Ruggiero, S. L., Dodson, T. B., Fantasia, J., Goodday, R., Aghaloo, T., Mehrotra, B., O'Ryan, F., \& American Association of Oral and Maxillofacial Surgeons (2014). American Association of Oral and Maxillofacial Surgeons position paper on medication-related osteonecrosis of the jaw--2014 update. Journal of oral and maxillofacial surgery: official journal of the American Association of Oral and Maxillofacial Surgeons, 72(10), $1938-1956$. https://doi.org/10.1016/j.joms.2014.04.031

Sarathy, A. P., Bourgeois, S. L., Jr, \& Goodell, G. G. (2005). Bisphosphonate-associated osteonecrosis of the jaws and endodontic treatment: two case reports. Journal of endodontics, 31(10), 759-763. https://doi.org/10.1097/01.don.0000182737.09980.2c

Vissink, A., Jansma, J., Spijkervet, F. K., Burlage, F. R., \& Coppes, R. P. (2003). Oral sequelae of head and neck radiotherapy. Critical reviews in oral biology and medicine: an official publication of the American Association of Oral Biologists, 14(3), 199-212. https://doi.org/10.1177/154411130301400305

Zecha, J. A., Raber-Durlacher, J. E., Nair, R. G., Epstein, J. B., Sonis, S. T., Elad, S., Hamblin, M. R., Barasch, A., Migliorati, C. A., Milstein, D. M., Genot, M. T., Lansaat, L., van der Brink, R., Arnabat-Dominguez, J., van der Molen, L., Jacobi, I., van Diessen, J., de Lange, J., Smeele, L. E., Schubert, M. M., \& Bensadoun, R. J. (2016). Low level laser therapy/photobiomodulation in the management of side effects of chemoradiation therapy in head and neck cancer: part 1: mechanisms of action, dosimetric, and safety considerations. Supportive care in cancer: official journal of the Multinational Association of Supportive Care in Cancer, 24(6), 2781-2792. https://doi.org/10.1007/s00520-016-3152-z 